\title{
The one-sided inverse along an element in semigroups and rings
}

\author{
Jianlong Chen*, Honglin Zou†, Huihui Zhu‡ Pedro Patrício ${ }^{\ddagger}$ \\ *Department of Mathematics, Southeast University, Nanjing 210096, China. \\ ${ }^{\dagger}$ School of Mathematics and Statistics, Hubei Normal University, Huangshi 435002, China. \\ ${ }^{\ddagger}$ School of Mathematics, Hefei University of Technology, Hefei 230009, China. \\ ${ }^{\S}$ CMAT-Centro de Matemática and Departamento de Matemática e Aplicacões, \\ Universidade do Minho, Braga 4710-057, Portugal.
}

\begin{abstract}
The concept of the inverse along an element was introduced by X. Mary in 2011. Later, H. H. Zhu etc. introduced the one-sided inverse along an element. In this paper, we first give a new existence criterion for the one-sided inverse along a product and characterize the existence of Moore-Penrose inverse by means of one-sided invertibility of certain element in a ring. In addition, we show that $a \in S^{\dagger} \bigcap S^{\#}$ if and only if $\left(a^{*} a\right)^{k}$ is invertible along $a$ if and only if $\left(a a^{*}\right)^{k}$ is invertible along $a$ in a $*$-monoid $S$, where $k$ is an arbitrary given positive integer. Finally, we prove that the inverse of $a$ along $a a^{*}$ coincides with core inverse of $a$ under the condition $a \in S^{\{1,4\}}$ in a $*$-monoid $S$.
\end{abstract}

Keywords: inverse along an element; von Neumann regularity; semigroups; rings

AMS Subject Classifications: 15A09; 16E50; 20M99; 16W99

\section{Introduction}

Throughout this paper, $S$ is a monoid (semigroup with identity) and $R$ is a ring with identity. We say $a$ is (von Neumann) regular in $S$ if there exists $x \in S$ such that $a x a=a$. Such $x$ is called an inner inverse of $a$ and denoted by $a^{-}$. An involution $*: \mathrm{S} \rightarrow \mathrm{S}$ is an anti-isomorphism which satisfies $(a b)^{*}=b^{*} a^{*}$ and $\left(a^{*}\right)^{*}=a$, where $a, b \in S$. *-monoid denotes the monoid with an involution.

Let us recall some definitions of generalized inverses. Let $S$ be a $*$-monoid, an element $a \in S$ is said to Moore-Penrose invertible if the following equations:

$$
\text { (1) } a x a=a, \quad(2) x a x=x, \quad(3)(a x)^{*}=a x, \quad(4)(x a)^{*}=x a
$$

\footnotetext{
${ }^{*}$ Corresponding author. E-mail: jlchen@seu.edu.cn

${ }^{\dagger}$ E-mail: honglinzou@163.com

${ }^{\ddagger}$ E-mail: ahzhh08@sina.com

§E-mail:pedro@math.uminho.pt
} 
has a common solution [12]. Such solution is unique if it exists, and is usually denoted by $a^{\dagger}$. The set of all Moore-Penrose invertible elements of $S$ will be denoted by $S^{\dagger}$. If $x \in S$ satisfies both (1) and (3), then $x$ is called an $\{1,3\}$-inverse of $a$ and denoted by $a^{(1,3)}$. The set of all $\{1,3\}$-invertible elements of $S$ will be denoted by $S^{\{1,3\}}$. Similarly, if $x \in S$ satisfies both (1) and (4), then $x$ is called an $\{1,4\}$-inverse of $a$ and denoted by $a^{(1,4)}$. The set of all $\{1,4\}$-invertible elements of $S$ will be denoted by $S^{\{1,4\}}$.

The Drazin inverse [2] of $a \in S$ is the element $x \in S$ which satisfies

$$
a^{k}=a^{k+1} x, \quad x a x=x, \quad a x=x a, \text { for some } k \geq 1 .
$$

The element $x$ above is unique if it exists and is denoted by $a^{D}$. The least such $k$ is called the index of $a$, and denoted by ind $(a)$. In particular, when $\operatorname{ind}(a)=1$, the Drazin inverse $a^{D}$ is called the group inverse of $a$ and it is denoted by $a^{\#}$. The set of all Drazin (resp. group) invertible elements of $S$ will be denoted by $S^{D}$ (resp. $\left.S^{\#}\right)$.

The core (resp. dual core) inverse [13] of $a \in R$ is the element $x \in R$ which satisfies

$$
a x a=a, \quad x R=a R(\operatorname{resp} . R x=R a), \quad R x=R a^{*}\left(\text { resp. } x R=a^{*} R\right) .
$$

The element $x$ above is unique if it exists and is denoted by $a^{\oplus}$ (resp. $\left.a_{\oplus}\right)$. The set of all core (resp. dual core) invertible elements of $R$ will be denoted by $R^{\oplus}$ (resp. $R_{\oplus}$ ).

In [9], X. Mary introduced a new generalized inverse using Green's preorders and relations [3], named the inverse along an element. The element $a \in S$ will be said to be invertible along $d \in S$ if there exists $b \in S$ such that

$$
b a d=d=d a b, \quad b S \subset d S, \quad S b \subset S d .
$$

If such $b$ exists, then it is unique and will be denoted by $a^{\| l d}$. This inverse unify some wellknown generalized inverse such as group inverse, Drazin inverse and Moore-Penrose inverse, that is $a^{\#}=a^{\| a}, a^{D}=a^{\| a^{k}}$ for some integer $m$ and $a^{\dagger}=a^{\| a^{*}}$.

In [10], X. Mary and P. Patrício gave a very useful existence criterion of $a^{\| d}$ by means of a unit in the ring, that is $a$ is invertible along $d$ if and only if $a d+1-d^{-} d$ is invertible if and only if $d a+1-d d^{-}$is invertible, when $d$ is regular.

In [14], H. H. Zhu etc. introduced left (right) invertible along an element. An element $a \in S$ is left (resp. right) invertible along $d \in S$ if there exists $b \in S$ such that

$$
b a d=d(\text { resp. } d a b=d), \quad S b \subset S d(\text { resp. } b S \subset d S) .
$$

They proved a surprising conclusion in a $*$-monoid $S, a \in S$ is left invertible along $a^{*}$ if and only if $a$ is right invertible $a^{*}$ if and only if $a$ is Moore-Penrose invertible.

In this paper, our motivation is that if $a$ is left (right) invertible along $d$, then we will consider that when $d$ is left (right) invertible along $a$ in a semigroup (or ring). For example, we can easily see that $a$ is invertible along $a^{*}$ if and only if $a^{*}$ is invertible along $a$ if and only if $a$ is Moore-Penrose invertible, where $a \in S, S$ is a $*$-monoid. 
In [14], the authors gave an existence criterion of the one-side inverse along $p m q$ (see [14, Theorem 3.2]). In addition, D. S. Rakić etc. [13] proved $a^{\oplus}=a^{\| a a^{*}}$ and $a_{\circledast}=a^{\| a^{*} a}$ under the condition $a \in R^{\dagger}$. According to these facts. In section 2, we further consider the inverse along a product $p m q$ and generalize some results of [14]. Conversely, we consider that $p m q$ is invertible along $a$. Also, we prove that a regular element $a \in R$ is Moore-Penrose invertible if and only if $\left(a a^{*}\right)^{k}+1-a a^{-}$is left invertible if and only $\left(a a^{*}\right)^{k}+1-a a^{-}$is right invertible, where $k$ is an arbitrary given positive integer. In section 3, we mainly obtain that $a \in S^{\dagger} \cap S^{\#}$ if and only if $\left(a^{*} a\right)^{k}$ is invertible along $a$ if and only if $\left(a a^{*}\right)^{k}$ is invertible along $a$, where $k$ is also an arbitrary given positive integer. In section 4 , we give that $a \in S^{\oplus}$ if and only if $a$ is invertible along $a a^{*}$ if and only if $a \in S^{\dagger} \cap S^{\sharp}$, under the condition $a \in S^{\{1,4\}}$.

Let $a \in R$, by $a_{l}^{-1}$ and $a_{r}^{-1}$ we denote a left inverse and a right inverse of $a$, respectively. First, we state some auxiliary results we will rely on.

Lemma 1.1. [8, Exercise 1.6] Let $a, b \in R$.

(1) If $1+a b$ is left invertible, then $1+b a$ is left invertible and $(1+b a)_{l}^{-1}=1-b(1+a b)_{l}^{-1} a$.

(2) If $1+a b$ is right invertible, then $1+b a$ is right invertible and $(1+b a)_{r}^{-1}=1-b(1+a b)_{r}^{-1} a$.

(3) If $1+a b$ is invertible, then $1+b a$ is invertible and $(1+b a)^{-1}=1-b(1+a b)^{-1} a$.

Lemma 1.2. Let $a, d \in S$. Then

(1) [14, Theorem 2.3] a is left invertible along $d$ if and only if $S d=S d a d$. In this case, $u d$ is a left inverse of a along $d$, where $d=u d a d, u \in S$.

(2) $[14$, Theorem 2.4] a is right invertible along $d$ if and only if $d S=d a d S$. In this case, $d v$ is a right inverse of a along $d$, where $d=d a d v, v \in S$.

(3) [10, Theorem 2.2] a is invertible along $d$ if and only if $S d=S d a d$ and $d S=d a d S$.

(4) $a$ is invertible along $d$ with inverse $y$ if and only if a is right invertible along $d$ with a right inverse $x$ and $a$ is left invertible along $d$ with a left inverse $z$. In this case $y=x=z$.

Proof. (4) We only need prove $y=x=z$. Suppose $a$ is invertible along $d$ with inverse $y$, then $y a d=d, S y \subset S d$. From $S y \subset S d$, it follows that there exists $t_{1} \in S$ such that $y=t_{1} d$. Since $x$ is a right inverse of $a$ along $d$, we get $d a x=d$ and $x S \subset d S$, which implies $x=d t_{2}$ for some $t_{2} \in S$. Hence, $y=t_{1} d=t_{1} d a x=y a x$, and $x=d t_{2}=y a d t_{2}=y a x$. So, $y=x$ holds. Similarly, we have $y=z$.

Lemma 1.3. Let $a, d \in R$ with $d$ regular. Then

(1) [14, Corollary 3.3] $a$ is left invertible along $d$ if and only if $u=d a+1-d d^{-}$is left invertible if and only if $v=a d+1-d^{-} d$ is left invertible. In this case, $u_{l}^{-1} d$ is a left inverse of a along $d$.

(2) [14, Corollary 3.5] a is right invertible along $d$ if and only if $u=d a+1-d d^{-}$is right invertible if and only if $v=a d+1-d^{-} d$ is right invertible. In this case, $d v_{r}^{-1}$ is a right inverse of a along $d$.

(3) [10, Theorem 3.2] $a$ is invertible along $d$ if and only if $u=d a+1-d d^{-}$is invertible if and only if $v=a d+1-d^{-} d$ is invertible. In this case, $a^{\| d}=u^{-1} d=d v^{-1}$. 
Lemma 1.4. [14, Theorem 2.16] Let $S$ be $a *$-monoid and let $a \in S$. Then a is Moore-Penrose invertible if and only if $a \in a a^{*} a S$ if and only if $a \in S a a^{*} a$.

Lemma 1.5. Let $S$ be $a *$-monoid and let $a \in S$.

(1) [14, Theorem 2.19] If $a=a a^{*} a x$ for some $x \in S$, then $a \in R^{\dagger}$ and $a^{\dagger}=a^{*} a x^{2} a^{*}$.

(2) $\left[14\right.$, Theorem 2.20] If $a=y a a^{*} a$ for some $y \in S$, then $a \in R^{\dagger}$ and $a^{\dagger}=a^{*} y^{2} a a^{*}$.

Lemma 1.6. [5, Theorem 1] Let $a \in S$. Then $a \in S^{\#}$ if and only if $a=a^{2} x=y a^{2}$ for some $x, y \in S$. In this case, $a^{\#}=y a x=y^{2} a=a x^{2}$.

Lemma 1.7. [4] Let $R$ be $a *$-ring and let $a, x, y \in R$. Then

(1) $x$ is $a\{1,3\}$-inverse of $a$ if and only if $a=x^{*} a^{*} a$.

(2) $y$ is $a\{1,4\}$-inverse of $a$ if and only if $a=a a^{*} y^{*}$.

Lemma 1.8. [7, Lemma 5.1] Let $R$ be $a$ *-ring and let $a \in R$. Then $a \in R^{\dagger}$ if and only if there exist $x, y \in R$ such that $a x a=a=a y a,(a x)^{*}=a x,(y a)^{*}=y a$. In this case, $a^{\dagger}=y a x$.

Next Lemma is proved in a $*$-ring (see [1, Proposition 2.1]). Indeed, it is true in a $*$-monoid.

Lemma 1.9. Let $S$ be $a *$-monoid and let $a \in S$. Then

(1) $a \in S^{\boxplus}$ if and only if $a \in S^{\sharp} \cap S^{\{1,3\}}$. In this case, $a^{\boxplus}=a^{\sharp} a a^{(1,3)}$.

(2) $a \in S_{\circledast}$ if and only if $a \in S^{\sharp} \cap S^{\{1,4\}}$. In this case, $a_{\circledast}=a^{(1,4)} a a^{\sharp}$.

\section{The one-sided inverse along the product $p m q$}

In this section, we give a new existence criterion for the one-side inverse along a product $p m q$ in a ring $R$, which covers [14, Theorem 3.2].

Theorem 2.1. Let $a, m, p, p^{\prime}, q, q^{\prime} \in R$ with $m$ regular and $k \geq 1$. If $p^{\prime} p m=m=m q q^{\prime}$, then the following are equivalent:

(1) a is left invertible along pmq;

(2) $u=(\text { qapm })^{k}+1-m^{-} m$ is left invertible;

(3) $v=(m q a p)^{k}+1-m m^{-}$is left invertible.

In this case, $p v_{l}^{-1}(m q a p)^{k-1} m q$ is a left inverse of a along pmq.

Proof. (2) $\Leftrightarrow(3)$ Since $u=(\text { qapm })^{k-1}$ qapm $+1-m^{-} m=1+\left((\text { qapm })^{k-1}\right.$ qap $\left.-m^{-}\right) m$, according to Lemma 1.1(1), we have $u$ is left invertible, i.e. $1+m\left((q a p m)^{k-1} q a p-m^{-}\right)=v$ is left invertible.

$(1) \Rightarrow(2)$ Suppose that $a$ is left invertible along $p m q$, by Lemma 1.2(1), we get $p m q=$ xpmqapmq for some $x \in R$. Multiplying the previous equality by $q^{\prime}$ from the right side and using the equality $m q q^{\prime}=m$, we have $p m=x p m q a p m$. Repeatedly use the equality 
$p m=x p m q a p m$, we have $p m=x($ pm $) q a p m=x(x p m q a p m) q a p m=x^{2} p m(q a p m)^{2}=\cdots=$ $x^{k}(p m)(q a p m)^{k}$. Then, note that $p^{\prime} p m=m$, we get

$$
\begin{aligned}
& \left(m m^{-} p^{\prime} x^{k} p m m^{-}+1-m m^{-}\right)\left(m(\text { qapm })^{k} m^{-}+1-m m^{-}\right) \\
= & m m^{-} p^{\prime}\left(x^{k} p m m^{-} m(q a p m)^{k}\right) m^{-}+1-m m^{-} \\
= & m m^{-} p^{\prime} p m m^{-}+1-m m^{-} \\
= & 1,
\end{aligned}
$$

which implies $m(\text { qapm })^{k} m^{-}+1-m m^{-}=1+m\left((\text { qapm })^{k}-1\right) m^{-}$is left invertible. Applying Lemma 1.1(1), we deduce that $1+\left((\text { qapm })^{k}-1\right) m^{-} m=(\text { qapm })^{k} m^{-} m+1-m^{-} m=(\text { qapm })^{k}+$ $1-m^{-} m=u$ is left invertible.

$(3) \Rightarrow(1)$ If $v=(m q a p)^{k}+1-m m^{-}$is left invertible, then there exists $s \in R$ such that $s\left((m q a p)^{k}+1-m m^{-}\right)=1$. Multiplying the previous equation by $m$ from the right side yields $m=s(m q a p)^{k} m$. Let $b=p s(m q a p)^{k-1} m q$, then $b a(p m q)=p s(m q a p)^{k-1} m q a p m q=$ $p s(m q a p)^{k} m q=p m q$. Since $p^{\prime} p m=m$, we get $b=p s(m q a p)^{k-1} m q=p s(m q a p)^{k-1} p^{\prime} p m q$, which implies $R b \subset R p m q$. Therefore, $b$ is a left inverse of $a$ along $p m q$.

As special cases of Theorem 2.1, we get the following results.

Corollary 2.2. [14, Theorem 3.2] Let $a, m, p, p^{\prime}, q, q^{\prime} \in R$ with $m$ regular. If $p^{\prime} p m=m=$ $m q q^{\prime}$, then the following are equivalent:

(1) a is left invertible along pmq;

(2) $u=q a p m+1-m^{-} m$ is left invertible;

(3) $v=$ mqap $+1-m^{-}$is left invertible.

In this case, $p v_{l}^{-1} m q$ is a left inverse of a along pmq.

Corollary 2.3. Let $a, m \in R$ with $m$ regular and $k \geq 1$. Then the following are equivalent:

(1) a is left invertible along $m$;

(2) $u=(a m)^{k}+1-m^{-} m$ is left invertible;

(3) $v=(m a)^{k}+1-m m^{-}$is left invertible.

In this case, $v_{l}^{-1}(m a)^{k-1} m$ is a left inverse of a along $m$.

Corollary 2.4. Let $a \in R$ be regular and $k \geq 1$. Then the following are equivalent:

(1) $R a=R a^{2}$;

(2) 1 is left invertible along a;

(3) $u=a^{k}+1-a^{-} a$ is left invertible;

(4) $v=a^{k}+1-a a^{-}$is left invertible.

In this case, $v_{l}^{-1} a^{k}$ is a left inverse of 1 along a.

Proof. (1) $\Leftrightarrow(2)$ By Lemma 1.2(1), we have (1) $\Leftrightarrow(2)$.

$(2) \Leftrightarrow(3) \Leftrightarrow(4)$ In Corollary 2.3, take $a=1, m=a$. then $(2) \Leftrightarrow(3) \Leftrightarrow(4)$.

Dually, we have the following results.

Theorem 2.5. Let $a, m, p, p^{\prime}, q, q^{\prime} \in R$ with $m$ regular and $k \geq 1$. If $p^{\prime} p m=m=m q q^{\prime}$, then the following are equivalent: 
(1) a is right invertible along $p m q$;

(2) $u=(\text { qapm })^{k}+1-m^{-} m$ is right invertible;

(3) $v=(m q a p)^{k}+1-m m^{-}$is right invertible.

In this case, $p m(q a p m)^{k-1} u_{r}^{-1} q$ is a right inverse of a along pmq.

Corollary 2.6. [14, Theorem 3.4] Let $a, m, p, p^{\prime}, q, q^{\prime} \in R$ with $m$ regular. If $p^{\prime} p m=m=$ $m q q^{\prime}$, then the following are equivalent:

(1) a is right invertible along pmq;

(2) $u=q a p m+1-m^{-} m$ is right invertible;

(3) $v=m q a p+1-m m^{-}$is right invertible.

In this case, $p m u_{r}^{-1} q$ is a right inverse of a along $p m q$.

Corollary 2.7. Let $a, m \in R$ with $m$ regular and $k \geq 1$. Then the following are equivalent:

(1) $a$ is right invertible along $m$;

(2) $u=(a m)^{k}+1-m^{-} m$ is right invertible;

(3) $v=(m a)^{k}+1-m m^{-}$is right invertible.

In this case, $m(a m)^{k-1} u_{r}^{-1}$ is a right inverse of a along $m$.

Corollary 2.8. Let $a \in R$ be regular and $k \geq 1$. Then the following are equivalent:

(1) $a R=a^{2} R$;

(2) 1 is right invertible along a;

(3) $u=a^{k}+1-a^{-} a$ is right invertible;

(4) $v=a^{k}+1-a a^{-}$is right invertible.

In this case, $a^{k} u_{r}^{-1}$ is a right inverse of $a^{k-1}$ along $a$.

According to Corollary 2.3, Corollary 2.7 and Lemma 1.2(4), we have the following result, which generalize [10, Theorem 3.2].

Corollary 2.9. Let $a, m \in R$ with $m$ regular and $k \geq 1$. Then the following are equivalent:

(1) $a$ is invertible along $m$;

(2) $u=(a m)^{k}+1-m^{-} m$ is invertible;

(3) $v=(m a)^{k}+1-m m^{-}$is invertible.

In this case, $a^{\| m}=v^{-1}(m a)^{k-1} m=m(a m)^{k-1} u^{-1}$.

We know that 1 is invertible $a$ if and only if $a \in R^{\#}$ (see [10, Corollary 3.4]). By Corollary 2.9 , we get

Corollary 2.10. Let $a \in R$ be regular and $k \geq 1$. Then the following are equivalent:

(1) $a \in R^{\#}$;

(2) $u=a^{k}+1-a^{-} a$ is invertible;

(3) $v=a^{k}+1-a a^{-}$is invertible.

In this case, $a^{\#}=a^{k} u^{-1}=v^{-1} a^{k}$. 
In [14], H. H. Zhu etc. showed $a \in R^{\dagger}$ if and only if $a$ is left(or right) invertible along $a^{*}$. In [11], P. Patrício proved that $a \in R^{\dagger}$ if and only if $a a^{*}+1-a a^{-}$is invertible if and only if $a^{*} a+1-a^{-} a$ is invertible. In the following theorem, we characterize the existence of $a^{\dagger}$ by means of one-side invertibility.

Theorem 2.11. Let $a \in R$ be regular and $k \geq 1$. Then the following are equivalent:

(1) a is Moore-Penrose invertible;

(2) $u=\left(a a^{*}\right)^{k}+1-a a^{-}$is left invertible;

(3) $u=\left(a a^{*}\right)^{k}+1-a a^{-}$is right invertible;

(4) $v=\left(a^{*} a\right)^{k}+1-a^{-} a$ is left invertible;

(5) $v=\left(a^{*} a\right)^{k}+1-a^{-} a$ is right invertible.

In this case,

$$
\begin{aligned}
a^{\dagger} & =a^{*}\left(u_{l}^{-1}\left(a a^{*}\right)^{k-1}\right)^{2} a a^{*}=a^{*} a\left(\left(a^{*} a\right)^{k-1} v_{r}^{-1}\right)^{2} a^{*} \\
& =a^{*}\left(a a^{*}\right)^{k-1}\left(u_{l}^{-1}\right)^{*}=\left(v_{r}^{-1}\right)^{*}\left(a^{*} a\right)^{k-1} a^{*} .
\end{aligned}
$$

Proof. (1) $\Leftrightarrow(2)$ Since a is regular, then $a^{*}$ is regular and $\left(a^{*}\right)^{-}=\left(a^{-}\right)^{*}$. In Corollary 2.7, let $m=a^{*}$. Then we have that $a$ is right invertible along $a^{*}$ if and only if $\left(a a^{*}\right)^{k}+1-\left(a^{*}\right)^{-} a^{*}=$ $\left(a a^{*}\right)^{k}+1-\left(a^{-}\right)^{*} a^{*}=\left(a a^{*}\right)^{k}+1-\left(a a^{-}\right)^{*}=u^{*}$ is right invertible. Note that $u$ is left invertible if and only if $u^{*}$ is right invertible. In addition, $\left(u^{*}\right)_{r}^{-1}=\left(u_{l}^{-1}\right)^{*}$. Thus, we get $(1) \Leftrightarrow(2)$. In this case, $a^{*}\left(a a^{*}\right)^{k-1}\left(u_{l}^{-1}\right)^{*}$ is a right inverse of $a$ along $a^{*}$. Applying Lemma 1.2(4), we get $a^{\dagger}=a^{*}\left(a a^{*}\right)^{k-1}\left(u_{l}^{-1}\right)^{*}$.

$(1) \Leftrightarrow(5)$ Similar to the proof of $(1) \Leftrightarrow(2)$. Also, we can have $a^{\dagger}=\left(v_{r}^{-1}\right)^{*}\left(a^{*} a\right)^{k-1} a^{*}$.

$(2) \Leftrightarrow(4)$ and $(3) \Leftrightarrow(5)$ Applying Lemma 1.1.

Next, we give the expression for $a^{\dagger}$. Since $u$ is left invertible, there exists $r \in R$ such that $r u=1$, which implies rua $=a$. Thus, $a=r u a=r\left(\left(a a^{*}\right)^{k}+1-a a^{-}\right) a=r\left(a a^{*}\right)^{k-1} a a^{*} a$, by Lemma 1.5(2), we get $a^{\dagger}=a^{*}\left(u_{l}^{-1}\left(a a^{*}\right)^{k-1}\right)^{2} a a^{*}$. Similarly, we can prove another expression for $a^{\dagger}$.

Take $k=1$ in Theorem 2.11, then we obtain the following corollary.

Corollary 2.12. Let $a \in R$ be regular. Then the following are equivalent:

(1) a is Moore-Penrose invertible;

(2) $u=a a^{*}+1-a a^{-}$is left invertible;

(3) $u=a a^{*}+1-a a^{-}$is right invertible;

(4) $v=a^{*} a+1-a^{-} a$ is left invertible;

(5) $v=a^{*} a+1-a^{-} a$ is right invertible.

In this case, $a^{\dagger}=a^{*} u_{l}^{-2} a a^{*}=a^{*} a v_{r}^{-2} a^{*}=a^{*}\left(u_{l}^{-1}\right)^{*}=\left(v_{r}^{-1}\right)^{*} a^{*}$.

In [9], X. Mary showed that $a \in R$ is invertible along $a^{k}$ if and only if $a$ is Drazin invertible. Naturally, we next consider when $a^{k}$ is invertible along $a$.

Theorem 2.13. Let $a \in S$ and $k \geq 0$. Then the following are equivalent:

(1) $a^{k}$ is left invertible along a;

(2) $S a=S a^{2}$. 
Proof. (1) $\Rightarrow(2)$ Suppose that $a^{k}$ is left invertible along $a$, by Lemma 1.2(1), we have $S a \subset$ $S a^{k} a^{2} \subset S a^{2}$, which implies $S a=S a^{2}$.

$(2) \Rightarrow(1)$ Assume $S a=S a^{2}$, then there exists $r \in S$ such that $a=r a^{2}$. Thus, $a=r a^{2}=$ $r^{2} a^{3}=\cdots=r^{k+1} a^{k+2} \in S a a^{k} a$. According to Lemma 1.2(1) again, we get $a^{k}$ is left invertible along $a$.

Dually, we have

Theorem 2.14. Let $a \in S$ and $k \geq 0$. Then the following are equivalent:

(1) $a^{k}$ is right invertible along $a$;

(2) $a S=a^{2} S$.

Using Theorem 2.13 and Theorem 2.14, we obtain

Corollary 2.15. Let $a \in S$ and $k \geq 0$. Then the following are equivalent:

(1) $a^{k}$ is invertible along a;

(2) $a \in S^{\#}$.

We next consider when the product paq is invertible along $d$ under certain condition.

Theorem 2.16. Let $a, d, p, p^{\prime}, q, q^{\prime} \in S$. If $q^{\prime} q d=d=d p p^{\prime}$, then the following are equivalent:

(1) paq is invertible along d with inverse $y$;

(2) $p a$ is right invertible along qd with a right inverse $x$ and aq is left invertible along $d p$ with a left inverse $z$.

In this case, $y=z a x$.

Proof. (1) $\Rightarrow(2)$ Suppose paq is invertible along $d$, by Lemma 1.2(3), we have $d p a q d S=d S$ and $S d p a q d=S d$, which imply $q d p a q d S=q d S$ and $S d p a q d p=S d p$. According to Lemma $1.2(1)(2)$, we have $p a$ is right invertible along $q d$ and $a q$ is left invertible along $d p$.

$(2) \Rightarrow(1)$ Suppose $p a$ is right invertible along $q d$ with a right inverse $x$, then $q d p a x=q d$ and $x S \subset q d S$. From $x S \subset q d S$, it follows that $x=q d t_{1}$ for suitable $t_{1} \in S$. Hence $q d p a q d t_{1}=$ $q d$. Multiplying the previous equation by $q^{\prime}$ from the left side, we get $q^{\prime} q d p a q d t_{1}=q^{\prime} q d$. Using the equation $q^{\prime} q d=d$, we obtain $d p a q d t_{1}=d$.

Similarly, since $a q$ is left invertible along $d p$ with a left inverse $z$, then $z a q d p=d p$ and $S z \subset S d p$. From $S z \subset S d p$, we get $z=t_{2} d p$ for some $t_{2} \in S$. Therefore, $t_{2} d p a q d p=d p$, which implies $t_{2} d p a q d p p^{\prime}=d p p^{\prime}$. Since $d p p^{\prime}=d$, then $t_{2} d p a q d=d$.

Let $u=z a x$. We will prove $u$ is the inverse of paq along $d$. Then, from above equations, we have

$$
\text { upaqd }=\text { zaxpaqd }=t_{2} d p a q d t_{1} p a q d=t_{2} d p a q d=d
$$

and

$$
d p a q u=d p a q z a x=d p a q t_{2} d p a q d t_{1}=d p a q d t_{1}=d .
$$

Also, $u=z a x=t_{2}\left(d p a q d t_{1}\right)=t_{2} d=\left(t_{2} d p a q d\right) t_{1}=d t_{1}$ implies $u S \subset d S$ and $S u \subset S d$. Thus, $u$ is the inverse of $p a q$ along $d$.

Note that, Theorem 2.16 is in general false without the condition $q^{\prime} q d=d=d p p^{\prime}$ : 
Example 2.17. Let $S$ be the algebra $M_{2}(\mathbb{F})$ of all $2 \times 2$ matrices over a field $\mathbb{F}$. Take

$$
p=a=q=\left[\begin{array}{ll}
1 & 0 \\
0 & 0
\end{array}\right], \quad d=\left[\begin{array}{ll}
1 & 0 \\
0 & 1
\end{array}\right] .
$$

Then, we can see that paq is not invertible, so paq is not invertible along d. However, pa ${ }^{\| d}=$ $a q^{\| d p}=a$.

\section{When $a^{*} a\left(\right.$ or $\left.a a^{*}\right)$ is invertible along $a$}

In this section, we mainly consider the relation between the (left, right) inverse of $a a^{*}\left(a^{*} a\right)$ along $a$ and the classical generalized inverses in a $*$-monoid. In what follows, $R$ always denotes a $*$-ring and $S$ denotes a $*$-monoid.

Theorem 3.1. Let $a \in S$ and $k \geq 1$. Then the following are equivalent:

(1) $a \in S^{\dagger}$ and $a S=a^{2} S$;

(2) $\left(a^{*} a\right)^{k}$ is right invertible along $a$.

Proof. (1) $\Rightarrow(2)$ From the condition $a \in S^{\dagger}$ and by Lemma 1.4, it follows that $a \in a a^{*} a S$, which imply $a=a a^{*} a h$ for some $h \in S$. Then, we have $a=a a^{*} a h=a\left(a^{*} a\right)^{2} h^{2}=\cdots=$ $a\left(a^{*} a\right)^{k} h^{k}$. According to the equality $a S=a^{2} S$, there exists $s \in S$ such that $a=a^{2} s$. Then, we have $a=a\left(a^{*} a\right)^{k} h^{k}=a\left(a^{*} a\right)^{k-1} a^{*} a h^{k}=a\left(a^{*} a\right)^{k-1} a^{*} a^{2} s h^{k}=a\left(a^{*} a\right)^{k} a s h^{k} \in a\left(a^{*} a\right)^{k} a S$. Applying Lemma 1.2(2), we can deduce that $\left(a^{*} a\right)^{k}$ is right invertible along $a$.

$(2) \Rightarrow(1)$ Suppose that $\left(a^{*} a\right)^{k}$ is right invertible along $a$, by Lemma 1.2(2), there exists $t \in S$ such that $a=a\left(a^{*} a\right)^{k} a t$ and hence $a^{*}=t^{*} a^{*}\left(a^{*} a\right)^{k} a^{*}$. Since $\left(a^{*} a\right)^{k} a t=a^{*} a\left(a^{*} a\right)^{k-1} a t=$ $t^{*} a^{*}\left(a^{*} a\right)^{k} a^{*} a\left(a^{*} a\right)^{k-1} a t=t^{*} a^{*}\left(a^{*} a\right)^{2 k} a t$, then we have $\left(\left(a^{*} a\right)^{k} a t\right)^{*}=\left(a^{*} a\right)^{k} a t$. Next, we will prove that $\left(a\left(a^{2} t\right)^{*}\right)^{*}=a\left(a^{2} t\right)^{*}$. Since

$$
\begin{aligned}
& a\left(a^{2} t\right)^{*} \\
= & a t^{*}\left(a^{2}\right)^{*}=a t^{*} a^{*} a^{*} \\
= & a t^{*} a^{*} t^{*} a^{*}\left(a^{*} a\right)^{k} a^{*} \\
= & a(\text { tat })^{*} a^{*}\left(a^{*} a\right)^{k-1} a^{*} a a^{*} \\
= & a(\text { tat })^{*} a^{*}\left(a^{*} a\right)^{k-1} a^{*} a\left(a^{*} a\right)^{k} a t a^{*} \\
= & a(\text { tat })^{*} a^{*}\left(a^{*} a\right)^{2 k-1} a^{*} a a t a^{*} \\
= & a(\text { tat })^{*} a^{*}\left(a^{*} a\right)^{2 k-1} a^{*} a\left(a^{*} a\right)^{k} \text { atata }^{*} \\
= & a(\text { tat })^{*} a^{*}\left(a^{*} a\right)^{3 k} a(\text { tat }) a^{*},
\end{aligned}
$$

it follows that $\left(a\left(a^{2} t\right)^{*}\right)^{*}=a\left(a^{2} t\right)^{*}$. Therefore, we get $a=a\left(a^{*} a\right)^{k} a t=a\left(\left(a^{*} a\right)^{k-1} a^{*} a^{2} t\right)^{*}=$ $a\left(a^{2} t\right)^{*} a\left(a^{*} a\right)^{k-1}=\left(a\left(a^{2} t\right)^{*}\right)^{*} a\left(a^{*} a\right)^{k-1}=a^{2} t\left(a^{*} a\right)^{k} \in a^{2} S$, which implies $a S=a^{2} S$.

Also, from the equality $a=a\left(a^{*} a\right)^{k} a t=a a^{*} a\left(a^{*} a\right)^{k-1} a t \in a a^{*} a S$, by Lemma 1.4, we deduce that $a \in S^{\dagger}$. 
Remark 3.2. Note that $a \in S^{\dagger}$ and $a S=a^{2} S$ can not imply $a \in S^{\#}$. For example, take $S$ to be the ring of both row-finite and column-finite infinite matrices over a field $\mathbb{F}$. Let involution * be the transpose. Take $a=\sum_{i=1}^{\infty} e_{i, i+1}$, where $e_{i, j}$ denotes the infinite matrix whose $(i, j)$-entry is 1 and others are zero. Then $a a^{*}=I$. Hence, we have $a^{\dagger}=a^{*}$, and $a S=a^{2} S$. However, $S a \neq S a^{2}$, which implies a is not group invertible.

Applying the previous theorem in a $*$-ring $R$, we have the following corollary.

Corollary 3.3. Let $a \in R$ be regular and $k \geq 1$. Then the following are equivalent:

(1) $a \in R^{\dagger}$ and $a R=a^{2} R$;

(2) $\left(a^{*} a\right)^{k}$ is right invertible along $a$;

(3) $u=a\left(a^{*} a\right)^{k}+1-a a^{-}$is right invertible;

(4) $v=\left(a^{*} a\right)^{k} a+1-a^{-} a$ is right invertible.

In this case, $a^{\dagger}=a^{*} a\left(\left(a^{*} a\right)^{k-1} a v_{r}^{-1}\right)^{2} a^{*}$.

Proof. (1) $\Leftrightarrow$ (2) By Theorem 3.1.

$(2) \Leftrightarrow(3)$ By Lemma 1.3.

$(3) \Leftrightarrow(4)$ By Lemma 1.1(2).

Next, we give the expression for the Moore-Penrose inverse $a^{\dagger}$. Since $v$ is right invertible, we have $v v_{r}^{-1}=1$, which implies $a=a\left(a^{*} a\right)^{k} a v_{r}^{-1}=a a^{*} a\left(a^{*} a\right)^{k-1} a v_{r}^{-1}$. By Lemma 1.5(1), we obtain $a^{\dagger}=a^{*} a\left(\left(a^{*} a\right)^{k-1} a v_{r}^{-1}\right)^{2} a^{*}$.

Dually, we have the following results.

Theorem 3.4. Let $a \in S$ and $k \geq 1$. Then the following are equivalent:

(1) $a \in S^{\dagger}$ and $S a=S a^{2}$;

(2) $\left(a a^{*}\right)^{k}$ is left invertible along a.

Corollary 3.5. Let $a \in R$ with a regular and $k \geq 1$. Then the following are equivalent:

(1) $a \in R^{\dagger}$ and $R a=R a^{2}$;

(2) $\left(a a^{*}\right)^{k}$ is left invertible along a;

(3) $u=a\left(a a^{*}\right)^{k}+1-a a^{-}$is left invertible;

(4) $v=\left(a a^{*}\right)^{k} a+1-a^{-} a$ is left invertible.

In this case, $a^{\dagger}=a^{*}\left(u_{l}^{-1} a\left(a a^{*}\right)^{k-1}\right)^{2} a a^{*}$.

In the following theorem, we consider when $a^{*} a$ (resp. $\left.a a^{*}\right)$ is left (resp. right) invertible along $a$ under the condition $a \in S^{\dagger}$.

Theorem 3.6. Let $a \in S^{\dagger}$ and $k \geq 1$. Then

(1) $S a=S a^{2}$ if and only if $\left(a^{*} a\right)^{k}$ is left invertible along $a$.

(2) $a S=a^{2} S$ if and only if $\left(a a^{*}\right)^{k}$ is right invertible along $a$. 
Proof. (1) Suppose $S a=S a^{2}$, we have $a=s a^{2}$ for some $s \in S$. According to the condition $a \in S^{\dagger}$ and Lemma 1.4, there exists $r \in S$ such that $a=r a a^{*} a$. Hence, we deduce that $a=s a^{2}=s\left(r a a^{*} a\right) a=s r r a a^{*} a a^{*} a a=s r^{2} a\left(a^{*} a\right)^{2} a=\cdots=s r^{k} a\left(a^{*} a\right)^{k} a \in S a\left(a^{*} a\right)^{k} a$. Вy Lemma 1.2(1), we get $\left(a^{*} a\right)^{k}$ is left invertible along $a$.

Conversely, suppose that $\left(a^{*} a\right)^{k}$ is left invertible along $a$. Using Lemma 1.2(1) again, there exists $t \in S$ such that $a=t a\left(a^{*} a\right)^{k} a=t\left(a a^{*}\right)^{k} a^{2}$, which implies $S a=S a^{2}$.

(2) This statement can be proved in the same manner as (1).

Note that, in the proof of sufficiency of Theorem 3.6, we need not $a \in S^{\dagger}$. So, we have the following questions.

Question 3.7. Suppose that $a^{*} a$ is left invertible along a, does $a \in S^{\dagger}$ hold? In addition, assume that a $a^{*}$ is right invertible along a, does $a \in S^{\dagger}$ hold?

We now give the relations of these inverses, such as the inverse of $a^{*} a$ along $a$, the inverse of $a a^{*}$ along $a$, Moore-Penrose inverse and group inverse.

Theorem 3.8. Let $a \in S$ and $k \geq 1$. Then the following are equivalent:

(1) $a \in S^{\dagger} \cap S^{\#}$

(2) $\left(a^{*} a\right)^{k}$ is right invertible along a and $\left(a a^{*}\right)^{k}$ is left invertible along a;

(3) $\left(a^{*} a\right)^{k}$ is invertible along a;

(4) $\left(a a^{*}\right)^{k}$ is invertible along a.

In this case,

$$
\begin{gathered}
a^{\dagger}=a^{*} a\left(\left(a^{*} a\right)^{k-1}\left(\left(a^{*} a\right)^{k}\right)^{\| a}\right)^{2} a^{*}=a^{*}\left(\left(\left(a a^{*}\right)^{k}\right)^{\| a}\left(a a^{*}\right)^{k-1}\right)^{2} a a^{*}, \\
a^{\#}=\left(\left(\left(a^{*} a\right)^{k}\right)^{\| a}\left(a^{*} a\right)^{k-1} a^{*}\right)^{2} a=a\left(a^{*}\left(a a^{*}\right)^{k-1}\left(\left(a a^{*}\right)^{k}\right)^{\| a}\right)^{2}, \\
\left(\left(a^{*} a\right)^{k}\right)^{\| a}=a a^{\#}\left(a^{\dagger}\left(a^{\dagger}\right)^{*}\right)^{k} \text { and }\left(\left(a a^{*}\right)^{k}\right)^{\| a}=\left(\left(a^{\dagger}\right)^{*} a^{\dagger}\right)^{k} a^{\#} a
\end{gathered}
$$

Proof. (1) $\Leftrightarrow(2)$ By Theorem 3.1 and 3.4.

$(1) \Rightarrow(3)$ According to the condition $a \in S^{\dagger} \cap S^{\#}$ and Theorem 3.6, we get $\left(a^{*} a\right)^{k}$ is left invertible along $a$. Applying Theorem $3.1,\left(a^{*} a\right)^{k}$ is right invertible along $a$. Hence, $\left(a^{*} a\right)^{k}$ is invertible along $a$.

$(3) \Rightarrow(2)$ Suppose that $\left(a^{*} a\right)^{k}$ is invertible along $a$, by Theorem 3.1, then $a \in S^{\dagger}$. Note that $\left(a^{*} a\right)^{k}$ is left invertible along $a$, by Lemma 1.2(1), we have $a \in S a\left(a^{*} a\right)^{k} a=S a\left(a^{*} a\right)^{k-1} a^{*} a^{2} \subset$ $S a^{2}$. By Theorem 3.4, we get $\left(a a^{*}\right)^{k}$ is left invertible along $a$.

$(1) \Rightarrow(4) \Rightarrow(2)$ It is similar to the proof of $(1) \Rightarrow(3) \Rightarrow(2)$.

Next, we give representations of $a^{\dagger}, a^{\#},\left(\left(a^{*} a\right)^{k}\right)^{\| a}$ and $\left(\left(a a^{*}\right)^{k}\right)^{\| a}$. Since $\left(a^{*} a\right)^{k}$ is invertible along $a$, we have

$$
a=a\left(a^{*} a\right)^{k}\left(\left(a^{*} a\right)^{k}\right)^{\| a}=a a^{*} a\left(a^{*} a\right)^{k-1}\left(\left(a^{*} a\right)^{k}\right)^{\| a}
$$

and

$$
a=\left(\left(a^{*} a\right)^{k}\right)^{\| a}\left(a^{*} a\right)^{k} a=\left(\left(a^{*} a\right)^{k}\right)^{\| a}\left(a^{*} a\right)^{k-1} a^{*} a^{2},
$$

which imply $a^{\dagger}=a^{*} a\left(\left(a^{*} a\right)^{k-1}\left(\left(a^{*} a\right)^{k}\right)^{\| a}\right)^{2} a^{*}$ and $a^{\#}=\left(\left(\left(a^{*} a\right)^{k}\right)^{\| a}\left(a^{*} a\right)^{k-1} a^{*}\right)^{2} a$ by Lemma 1.5 and Lemma 1.6, respectively.

Similarly, we get $a^{\dagger}=a^{*}\left(\left(\left(a a^{*}\right)^{k}\right)^{\| a}\left(a a^{*}\right)^{k-1}\right)^{2} a a^{*}$ and $a^{\#}=a\left(a^{*}\left(a a^{*}\right)^{k-1}\left(\left(a a^{*}\right)^{k}\right)^{\| a}\right)^{2}$. 
Note that $a=a\left(a^{*} a\right)^{k} a a^{\#}\left(a^{\dagger}\left(a^{\dagger}\right)^{*}\right)^{k}$, by Lemma 1.2, we have $\left(\left(a^{*} a\right)^{k}\right)^{\| a}=a a^{\#}\left(a^{\dagger}\left(a^{\dagger}\right)^{*}\right)^{k}$. Similarly, from $a=\left(\left(a^{\dagger}\right)^{*} a^{\dagger}\right)^{k} a^{\#} a\left(a a^{*}\right)^{k} a$, it follows that $\left(\left(a a^{*}\right)^{k}\right)^{\| a}=\left(\left(a^{\dagger}\right)^{*} a^{\dagger}\right)^{k} a^{\#} a$.

Letting $k=1$ in Theorem 3.8, we get

Corollary 3.9. Let $a \in S$. Then the following are equivalent:

(1) $a \in S^{\dagger} \cap S^{\#}$;

(2) $a^{*} a$ is right invertible along $a$ and $a a^{*}$ is left invertible along $a$;

(3) $a^{*} a$ is invertible along $a$;

(4) $a a^{*}$ is invertible along $a$.

In this case,

$$
\begin{gathered}
a^{\dagger}=a^{*} a\left(\left(a^{*} a\right)^{\| a}\right)^{2} a^{*}=a^{*}\left(\left(a a^{*}\right)^{\| a}\right)^{2} a a^{*}, \\
a^{\#}=\left(\left(a^{*} a\right)^{\| a} a^{*}\right)^{2} a=a\left(a^{*}\left(a a^{*}\right)^{\| a}\right)^{2} \\
\left(a^{*} a\right)^{\| a}=a^{\#}\left(a^{\dagger}\right)^{*} \text { and }\left(a a^{*}\right)^{\| a}=\left(a^{\dagger}\right)^{*} a^{\#} .
\end{gathered}
$$

Applying Theorem 3.8, Lemma 1.3 and Lemma 1.9 in a $*$-ring $R$, we have the following corollary.

Corollary 3.10. Let $a \in R$ be regular and $k \geq 1$. Then the following are equivalent:

(1) $a \in R^{\dagger} \cap R^{\#}$

(2) $a \in R^{\oplus} \bigcap R_{\oplus}$;

(3) $u=a\left(a^{*} a\right)^{k}+1-a a^{-}$is invertible;

(4) $v=\left(a a^{*}\right)^{k} a+1-a^{-} a$ is invertible;

(5) $s=\left(a^{*} a\right)^{k} a+1-a^{-} a$ is invertible;

(6) $t=a\left(a a^{*}\right)^{k}+1-a a^{-}$is invertible.

In this case,

$$
\begin{aligned}
a^{\boxplus} & =u^{-1} a\left(a^{*} a\right)^{k-1} a^{*}, a_{\circledast}=a^{*}\left(a a^{*}\right)^{k-1} a v^{-1}, \\
a^{\dagger} & =\left(t^{-1} a\left(a a^{*}\right)^{k-1} a\right)^{*}=\left(a\left(a^{*} a\right)^{k-1} a s^{-1}\right)^{*}
\end{aligned}
$$

and

$$
a^{\#}=\left(u^{-1} a\left(a^{*} a\right)^{k-1} a^{*}\right)^{2} a=a\left(a^{*}\left(a a^{*}\right)^{k-1} a v^{-1}\right)^{2} .
$$

Proof. We only need to prove the expressions of $a^{\boxplus}, a_{\oplus}, a^{\dagger}$ and $a^{\#}$. Observe that $u a=$ $a\left(a^{*} a\right)^{k} a=a\left(a^{*} a\right)^{k-1} a^{*} a^{2}$, which implies $a=u^{-1} a\left(a^{*} a\right)^{k-1} a^{*} a^{2}$. Since $a \in R^{\#}$, by Lemma 1.6, we have $a^{\#}=\left(u^{-1} a\left(a^{*} a\right)^{k-1} a^{*}\right)^{2} a$. Using Lemma 1.9 , we obtain

$$
\begin{aligned}
a^{\boxplus} & =a^{\#} a a^{(1,3)}=\left(u^{-1} a\left(a^{*} a\right)^{k-1} a^{*}\right)^{2} a^{2} a^{(1,3)} \\
& =u^{-1} a\left(a^{*} a\right)^{k-1} a^{*}\left(u^{-1} a\left(a^{*} a\right)^{k-1} a^{*} a^{2}\right) a^{(1,3)} \\
& =u^{-1} a\left(a^{*} a\right)^{k-1} a^{*} a a^{(1,3)} \\
& =u^{-1} a\left(a^{*} a\right)^{k-1} a^{*} .
\end{aligned}
$$

Similarly, we can get $a^{\#}=a\left(a^{*}\left(a a^{*}\right)^{k-1} a v^{-1}\right)^{2}$ and $a_{\circledast}=a^{*}\left(a a^{*}\right)^{k-1} a v^{-1}$. 
From $a s=a\left(a^{*} a\right)^{k} a$ and $t a=a\left(a a^{*}\right)^{k} a$, it follows that $a=a a^{*} a\left(a^{*} a\right)^{k-1} a s^{-1}$ and $a=$ $t^{-1} a\left(a a^{*}\right)^{k-1} a a^{*} a$. Applying Lemma 1.7 and Lemma 1.8, we have

$$
\begin{aligned}
a^{\dagger} & =\left(a\left(a^{*} a\right)^{k-1} a s^{-1}\right)^{*} a\left(t^{-1} a\left(a a^{*}\right)^{k-1} a\right)^{*} \\
& =\left(s^{-1}\right)^{*} a^{*}\left(a^{*} a\right)^{k-1} a^{*} a a^{*}\left(a a^{*}\right)^{k-1} a^{*}\left(t^{-1}\right)^{*} \\
& =\left(s^{-1}\right)^{*}\left(a\left(a^{*} a\right)^{k} a\right)^{*}\left(a a^{*}\right)^{k-1} a^{*}\left(t^{-1}\right)^{*} \\
& =\left(s^{-1}\right)^{*}(a s)^{*}\left(a a^{*}\right)^{k-1} a^{*}\left(t^{-1}\right)^{*} \\
& =a^{*}\left(a a^{*}\right)^{k-1} a^{*}\left(t^{-1}\right)^{*} \\
& =\left(t^{-1} a\left(a a^{*}\right)^{k-1} a\right)^{*} .
\end{aligned}
$$

Also, we can have $a^{\dagger}=\left(a\left(a^{*} a\right)^{k-1} a s^{-1}\right)^{*}$.

\section{When $a$ is invertible along $a a^{*}$ ( or $a^{*} a$ )}

In [13], D. S. Rakić etc. showed that the inverse of $a$ along $a a^{*}$ coincides with core inverse of $a$, under the condition $a \in R^{\dagger}$. Next, we will consider these kinds of inverses under weaker condition in a $*$-monoid.

It is well known that $a \in S^{\{1,4\}}$ if and only if $a \in a a^{*} S$. Under the hypothesis $a \in S^{\{1,4\}}$, we discuss the relation between the one-side inverse of $a$ along $a a^{*}$ and the one-side inverse of $a^{*} a$ along $a$.

Theorem 4.1. Let $a \in S^{\{1,4\}}$. Then the following are equivalent:

(1) $a$ is left invertible along $a a^{*}$;

(2) $a^{*} a$ is left invertible along a.

Proof. (1) $\Rightarrow(2)$ Suppose that $a$ is left invertible along $a a^{*}$, by Lemma 1.2(1), we have $a a^{*} \in$ $S a a^{*} a^{2} a^{*}$, which implies $a a^{*}=t_{1} a a^{*} a^{2} a^{*}$ for some $t_{1} \in S$. From the condition $a \in S^{\{1,4\}}$, there exists $t_{2} \in S$ such that $a=a a^{*} t_{2}$. Hence, we deduce that $a=a a^{*} t_{2}=t_{1} a a^{*} a^{2} a^{*} t_{2}=t_{1} a a^{*} a^{2}$. According to Lemma 1.2(1) again, we get $a^{*} a$ is left invertible along $a$.

$(2) \Rightarrow(1)$ Since $a^{*} a$ is left invertible along $a$, by Lemma $1.2(1)$, we have $a=t_{3} a a^{*} a^{2}$ for some $t_{3} \in S$. Multiplying the previous equation by $a^{*}$ from the right side yields $a a^{*}=$ $t_{3} a a^{*} a^{2} a^{*}$. Hence, $a$ is left invertible along $a a^{*}$.

Corollary 4.2. Let $a \in R^{\{1,4\}}$. Then the following are equivalent:

(1) $a$ is left invertible along $a a^{*}$;

(2) $u=a a^{*} a+1-a a^{(1,4)}$ is left invertible;

(3) $v=a^{*} a^{2}+1-a^{(1,4)} a$ is left invertible;

(4) $f=\left(a^{*}\right)^{2} a+1-a^{(1,4)} a$ is right invertible;

(5) $g=a\left(a^{*}\right)^{2}+1-a a^{(1,4)}$ is right invertible.

In this case, $u_{l}^{-1} a a^{*}$ is a left inverse of a along a $a^{*}$.

Proof. (1) $\Leftrightarrow(2)$ Since $a \in R^{\{1,4\}}$ and by Lemma 1.3(1), we have $a^{*} a$ is left invertible along $a$ if and only if $a a^{*} a+1-a a^{(1,4)}$ is left invertible. By Theorem 4.1, it follows that $(1) \Leftrightarrow(2)$. 
(3) $\Leftrightarrow(4)$ Note that $v=f^{*}$, then we get $(3) \Leftrightarrow(4)$.

$(2) \Leftrightarrow(3)$ and $(4) \Leftrightarrow(5)$ By Lemma 1.1(1)(2).

Suppose that $u$ is left invertible, then $u_{l}^{-1} u=1$, which implies $u_{l}^{-1} u a a^{*}=a a^{*}$. Note that $a a^{*}=u_{l}^{-1} u a a^{*}=u_{l}^{-1}\left(a a^{*} a+1-a a^{(1,4)}\right) a a^{*}=u_{l}^{-1} a a^{*} a a a^{*}$. Hence, $u_{l}^{-1} a a^{*}$ is a left inverse of $a$ along $a a^{*}$ by Lemma 1.2(1).

Similarly, we have the following results.

Theorem 4.3. Let $a \in S^{\{1,4\}}$. Then the following are equivalent:

(1) a is right invertible along $a a^{*}$;

(2) $a^{*} a$ is right invertible along $a$.

Proof. Since $a \in S^{\{1,4\}}$, there exists $t_{2} \in S$ such that $a=a a^{*} t_{2}$.

$(1) \Rightarrow(2)$ Note that $a a^{*}=a a^{*} a^{2} a^{*} t_{1}$ for some $t_{1} \in S$ by Lemma 1.2(2). Thus, $a=a a^{*} t_{2}=$ $a a^{*} a^{2} a^{*} t_{1} t_{2} \in a\left(a^{*} a\right) a S$, which implies $a^{*} a$ is right invertible along $a$.

$(2) \Rightarrow(1)$ Suppose that $a^{*} a$ is right invertible along $a$, there exists $t_{3} \in S$ such that $a=a\left(a^{*} a\right) a t_{3}=a a^{*} a\left(a a^{*} t_{2}\right) t_{3}$. Then $a a^{*}=\left(a a^{*}\right) a\left(a a^{*}\right) t_{2} t_{3} a^{*} \in\left(a a^{*}\right) a\left(a a^{*}\right) S$, which gives $a$ is right invertible along $a a^{*}$.

Corollary 4.4. Let $a \in R^{\{1,4\}}$. Then the following are equivalent:

(1) $a$ is right invertible along $a a^{*}$;

(2) $u=a a^{*} a+1-a a^{(1,4)}$ is right invertible;

(3) $v=a^{*} a^{2}+1-a^{(1,4)} a$ is right invertible;

(4) $f=\left(a^{*}\right)^{2} a+1-a^{(1,4)} a$ is left invertible;

(5) $g=a\left(a^{*}\right)^{2}+1-a a^{(1,4)}$ is left invertible.

In this case, $a a^{*}\left(g_{l}^{-1}\right)^{*}$ is a right inverse of a along $a a^{*}$.

Theorem 4.5. Let $a \in S^{\{1,4\}}$. Then the following are equivalent:

(1) $a$ is invertible along $a a^{*}$;

(2) $a^{*} a$ is invertible along $a$;

(3) $a \in S^{\dagger} \cap S^{\sharp}$;

(4) $a \in S^{\oplus}$.

In this case, $a^{\oplus}=a^{\| a a^{*}}$.

Proof. (1) $\Leftrightarrow(2)$ According to Theorem 4.1 and Theorem 4.3, we have (1) $\Leftrightarrow(2)$.

$(2) \Leftrightarrow(3)$ The equivalence of (2) and (3) can be obtained by Corollary 3.9.

$(3) \Leftrightarrow(4)$ Using Lemma 1.9 and $a \in S^{\{1,4\}}$, we have $(3) \Leftrightarrow(4)$.

Next, we will prove the inverse of $a$ along $a a^{*}$ coincides with core inverse of $a$ under the condition $a \in S^{\{1,4\}}$. Since $a^{\boxplus}=a^{\sharp} a a^{(1,3)}$, we have $a^{\boxplus} a\left(a a^{*}\right)=a^{\sharp} a a^{(1,3)} a\left(a a^{*}\right)=a a^{*}$ and $a^{\boxplus}=a^{\sharp} a a^{(1,3)}=a^{\#}\left(a^{(1,3)}\right)^{*} a^{(1,4)} a a^{*} \in S a a^{*}$, which imply $a^{\boxplus}$ is a left inverse of $a$ along $a a^{*}$. According to Lemma $1.2(4)$, we have $a^{\boxplus}=a^{\| a a^{*}}$.

Remark 4.6. Note that $a$ is invertible along aa* can not imply $a \in S^{\#}$ or $a \in S^{\{1,3\}}$ or $a \in S^{\{1,4\}}$. For example, let $S=Z_{4}$ and $x^{*}=x$ for any $x \in S$. Take $a=2$, then $a a^{*}=0$ and $a^{\| a a^{*}}=0$. But $a$ is not regular, so $a \notin S^{\#}, a \notin S^{\{1,3\}}$ and $a \notin S^{\{1,4\}}$. 
Remark 4.7. Under the condition $a \in S^{\dagger}$, we can not have the conclusion a is left(right) invertible along a $a^{*}$. For example, let $S=M_{2}(\mathbb{H})$ and the involution be the conjugate transpose, where $\mathbb{H}$ denotes the division ring of quaternions. We know that any element in $S$ is MoorePenrose invertible. Take $a=\left[\begin{array}{cc}i-j & 1-k \\ 1+k & -i-j\end{array}\right]$. Then $d=: a a^{*}=4\left[\begin{array}{cc}1 & i \\ -i & 1\end{array}\right]$, $a a^{*} a=8 a$ and $d a d=a a^{*} a a a^{*}=8 a a a^{*}=0$. Hence, $d \notin S d a d(d \notin d a d S)$, which imply $a$ is not left(right) invertible along $a a^{*}$.

Remark 4.8. We have seen that a is left invertible along $a^{*}$ if and only if a is right invertible along $a^{*}$. However, the following example shows that $a$ is left invertible along a $a^{*}$ is not equivalent to a is right invertible along a $a^{*}$ in general.

Example 4.9. Let $S$ be the ring which is the same as the infinite matrix ring in Remark 3.2 and let $a=\sum_{i=1}^{\infty} e_{i+1, i}$. Then, $d=: a a^{*}=\sum_{i=2}^{\infty} e_{i, i}$ and $d a d=\sum_{i=2}^{\infty} e_{i+1, i}$. We can easily see that $d \notin d a d S$, which implies a is not right invertible along $d$. While, $d=\left(\sum_{i=2}^{\infty} e_{i, i+1}\right) d a d \in S d a d$, we deduce that a is left invertible along $d$.

Remark 4.10. In Theorem 4.5, we can not replace $a \in S^{\{1,4\}}$ with $a \in S^{\{1,3\}}$. For example, let $S=M_{2}(\mathbb{C})$ and the involution is the transpose. Take $a=\left[\begin{array}{cc}1 & i \\ 0 & 0\end{array}\right]$. Then $a \in S a^{*} a$, which implies $a \in S^{\{1,3\}}$. Note that $a a^{*}=0$, a is invertible along $a a^{*}$. But, $a \notin a a^{*} S$, which yields $a \notin S^{\{1,4\}}$ and $a \notin S^{\dagger}$.

Similar to Theorem 4.5, we have the following result.

Theorem 4.11. Let $a \in S^{\{1,3\}}$. Then the following are equivalent:

(1) $a$ is invertible along $a^{*} a$;

(2) $a a^{*}$ is invertible along $a$;

(3) $a \in S^{\dagger} \cap S^{\sharp}$;

(4) $a \in S_{\oplus}$.

In this case, $a_{\circledast}=a^{\| a^{*} a}$.

According to Theorem 4.5 and Theorem 4.11, we get

Corollary 4.12. [13, Theorem 4.3] Let $a \in R^{\dagger}$. Then

(1) $a$ is core invertible if and only if a is invertible along a $a^{*}$. In this case, the inverse of a along a $a^{*}$ coincides with core inverse of $a$.

(2) $a$ is dual core invertible if and only if $a$ is invertible along $a^{*} a$. In this case, the inverse of a along $a^{*} a$ coincides with dual core inverse of $a$.

Acknowledgements This research was supported by the National Natural Science Foundation of China (No. 11371089), the Specialized Research Fund for the Doctoral Program of Higher Education (No. 20120092110020), the Natural Science Foundation of Jiangsu Province (No. BK20141327) and the Foundation of Graduate Innovation Program of Jiangsu Province (No. KYZZ15-0049). 


\section{References}

[1] J. L. Chen, H. H. Zhu. P. Patrício, Y. L. Zhang, Characterizations and representations of core and dual core inverses in rings. Manuscript (2016).

[2] M. P. Drazin, Pseudo-inverse in associative rings and semigroups, Amer. Math. Monthly 65 (1958) 506-514.

[3] J. A. Green, On the structure of semigroups, Ann. Math. 54 (1951) 163-172.

[4] R. E. Hartwig. Block generalized inverses, Arch. Ration. Mech. Anal. 61 (1976) 197-251.

[5] R. E. Hartwig, J. Luh, A note on the group structure of unit regular ring elements, Pacific J. Math. 71 (1977) 449-461.

[6] J. J. Koliha, D. Djordjević, D. Cvetković, Moore-Penrose inverse in rings with involution, Linear Algebra Appl. 426 (2007) 371-381.

[7] J. J. Koliha, P. Patrício, Elements of rings with equal spectral idempotents, J. Austral. Math. Soc. 72 (2002) 137-152.

[8] T. Y. Lam, A First Course in Noncommutative Rings, Second ed., Grad. Text in Math., Vol. 131, Springer-Verlag, Berlin-Heidelberg-New York, 2001.

[9] X. Mary, Reprint of: On generalized inverses and Green's relations, Linear Algebra Appl. 438 (2013) 1532-1540.

[10] X. Mary, P. Patrício, Generalized inverses modulo $\mathcal{H}$ in semigroups and rings, Linear Multilinear Algebra 61 (8) (2013) 1130-1135.

[11] P. Patrício, The Moore-Penrose inverse of von Neumann regular matrices over a ring, Linear Algebra Appl. 332/334 (2001) 469-483.

[12] R. Penrose, A generalized inverse for matrices, Proc. Cambridge Philos. Soc. 51 (1955) 406-413.

[13] D. S. Rakić, N. Č. Dinčić, D. S. Djordjević, Group, Moore-Penrose, core and dual core inverse in rings with involution, Linear Algebra Appl. 463 (2014) 115-133.

[14] H. H. Zhu, J. L. Chen and P. Patrício, Further results on the inverse along an element in semigroups and rings, Linear Multilinear Algebra 64 (3) (2016) 393-403.

[15] H. H. Zhu, P. Patrício, J. L. Chen and Y. L. Zhang, The inverse along a product and its applications, Linear Multilinear Algebra, DOI: 10.1080/03081087.2015.1059796. 